\title{
Multi-rogue waves solutions to the focusing NLS equation and the KP-I equation
}

\author{
P. Dubard and V. B. Matveev \\ IMB, Université de Bourgogne, 9 av. Alain Savary, Dijon, France
}

Received: 29 September 2010 - Accepted: 20 December 2010 - Published: 3 March 2011

\begin{abstract}
We construct a multi-parametric family of quasi-rational solutions to the focusing NLS equation, presenting a profile of multiple rogue waves. These solutions have also been used by us to construct a large family of smooth, real localized rational solutions of the KP-I equation quite different from the multi-lumps solutions first constructed in Bordag et al. (1977). The physical relevance of both equations is very large. From the point of view of geosciences, the focusing NLS equation is relevant to the description of surface waves in deep water, and the KP-I equation occurs in the description of capillary gravitational waves on a liquid surface, but also when one considers magneto-acoustic waves in plasma (Zhdanov, 1984 ) etc. In addition, there are plenty of equations of physical importance, having their origin in fiber optics, hydrodynamics, plasma physics and many other areas, which are gauge equivalent to the NLS equation or to the KP-I equation. Therefore our results can be easily extended to a large number of systems of physical interest to be discussed in separate publications.
\end{abstract}

\section{Introduction}

Roughly speaking, in oceanography a rogue wave is a unexpectedly high wave strongly localized in space-time although recently, certain authors have also speculated about "long-life" rogue waves. Even if testimonies about such freak phenomena have been available for a long time, the study of rogue waves has been booming for a couple of decades, following the first scientific recording of an appearance of a rogue wave in the ocean. A very good review of the "state of the art" before 2009 can be found in

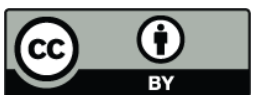

Correspondence to: P. Dubard (philippe.dubard@u-bourgogne.fr)
Kharif et al. (2009). Since then, the notion of rogue waves has appeared in several other fields such as nonlinear optics where a Peregrine breather has been observed recently for the first time (Kibler et al., 2010). In all cases, one of the simplest models used is the focusing NLS equation

$i v_{t}+v_{x x}+2|v|^{2} v=0$.

Here we discuss an important class of solutions of (1), representing the rational $2 n$-parametric modulation of the plane wave solution of fixed amplitude $B$. This class of solutions was first constructed in 1986 in the article by Eleonskii et al. (1986).

Its relevance to the multi-rogue wave solutions was understood only 24 years later in our work (Dubard et al., 2010; Matveev et al., 2010) providing also a simplified derivation of the results of Eleonskii et al. (1986) using Darboux transform (see Matveev et al., 1991). In our work, the connection of these solutions to a class of $2 n$-parametric family of smooth rational real solutions to the KP-I equation was also first discovered.

The case $n=1$ reproduces a famous Peregrine breather solution of the focusing NLS equation. Its amplitude reaches one high local maximum on $(x, t)$ plane. For $n=2$, in general, the amplitude of these solutions attends 3 big maxima of the height several times higher with respect to the amplitude of the background plane wave solution. We conjecture that for higher values of $n$ "in general position" the number of these maxima is equal to $n(n+1) / 2$. This conjecture is supported by the tested solutions corresponding to $n=3$ and $n=4$. Indeed this is true for all tested "generic" values of parameters. In some exceptional cases corresponding to the "higher order" Peregrine breathers constructed in (Akhmediev et al., 2009a,b,c, 2010), the number of local maximums for $n=2$ is equal 5 and one of them is much higher than others. All the constructed solutions have exactly the behavior of waves "appearing from nowhere and disappearing again".

Published by Copernicus Publications on behalf of the European Geosciences Union. 
We also discuss the related family of smooth real rational solutions of the KP-I equation. Their qualitative analysis is still far from being completed but already we have a reason to consider some of the related solutions as two-dimensional rogue waves.

\section{Multi-rogue waves solutions to the focusing NLS equation}

\subsection{Some notations and definitions}

Below we describe some auxiliary objects allowing the construction of a family of nonsingular (for all real $x$ and $t$ ) quasi-rational solutions of the focusing NLS equation having the property to reach a maximum of the amplitude at some fixed points of $(x, t)$-plane and so that when $t^{2}+x^{2} \rightarrow \infty$,

$|v|^{2} \rightarrow B^{2}$

where $B$ is any arbitrary chosen real constant.

Let $q_{2 n}(k)$ be a polynomial of degree $2 n$ defined by the formula

$$
\begin{aligned}
q_{2 n}(k) & :=\prod_{j=1}^{n}\left(k^{2}-\frac{\omega^{2 m_{j}+1}+1}{\omega^{2 m_{j}+1}-1} B^{2}\right), \\
\omega & :=\exp \left(\frac{i \pi}{2 n+1}\right),
\end{aligned}
$$

where $m_{j}$ are positive integers satisfying the condition

$0 \leq m_{j} \leq 2 n-1, \quad m_{l} \neq 2 n-m_{j}$,

for all $l$ and $j$. $^{1}$

We use also the definitions:

$$
\begin{aligned}
\Phi(k) & :=i \sum_{l=1}^{2 n} \varphi_{l}(i k)^{l}, \varphi_{j} \in \mathbf{R}, \\
f(k, x, t) & :=\frac{\exp \left(k x+i k^{2} t+\Phi(k)\right)}{q_{2 n}(k)}, D_{k}:=\frac{k^{2}}{k^{2}+B^{2}} \frac{\partial}{\partial k}, \\
f_{j}(x, t) & :=\left.D_{k}^{2 j-1} f(k, x, t)\right|_{k=B}, \\
f_{n+j}(x, t) & :=\left.D_{k}^{2 j-1} f(k, x, t)\right|_{k=-B}, B \in \mathbf{R}, j=1, \ldots, n .
\end{aligned}
$$

Below we use the standard notation $W\left(g_{1}, \ldots, g_{m}\right)$ for a Wronskian determinant of any $m$ functions:

$W\left(g_{1}, \ldots, g_{m}\right):=\operatorname{det} A, \quad A_{p j}=\partial_{x}^{p-1} g_{j}, \quad p, j=1, \ldots m$.

We denote $W_{1}$ and $W_{2}$ two Wronskian determinants composed from the functions $f$ and $f_{j}$ defined above:

$$
\begin{aligned}
& W_{1}:=W\left(f_{1}, \ldots, f_{2 n}\right), \\
& W_{2}:=W\left(f_{1}, \ldots, f_{2 n}, f\right) .
\end{aligned}
$$

\footnotetext{
${ }^{1}$ For instance it is possible to take $m_{j}=j-1$.
}

\subsection{Multi-rogue solutions to the focusing NLS equation: the main theorem}

Theorem 1. The function $v(x, t)$ defined by the formula

$v(x, t)=-q_{2 n}(0) B^{1-2 n} e^{2 i B^{2} t} \frac{\left.W_{2}\right|_{k=0}}{W_{1}}$

represents a family of nonsingular quasi-rational solutions of (1) depending on $2 n+1$ independent real parameters $B, \varphi_{j}$, $j=1, \ldots, 2 n$. When $t^{2}+x^{2} \rightarrow \infty,|v|^{2} \rightarrow B^{2}$.

The formulation above is equivalent to the main result of Eleonskii et al. (1986) written in more elegant notations. ${ }^{2}$ Without loss of generality we can take $B=1$ since the NLS equation is invariant with respect to the scaling transformation $v(x, t) \rightarrow B v\left(B x, B^{2} t\right)$. Therefore, below we always set $B=1$. As mentioned before, the simplest case $n=1$, when we have only 3 real parameters $\left(\varphi_{1}, \varphi_{2}, B\right)$ reproduces the Peregrine breather, and $\varphi_{1,2}$ are translation parameters. For $\mathrm{B}=1$ its analytical form is given by the formula

$$
\begin{aligned}
& v(x, t) \\
& =\frac{\left(x-\varphi_{1}\right)^{2}+4\left(t-\varphi_{2}\right)^{2}-(2 \sqrt{3}+4 i)\left(t-\varphi_{2}\right)+i \sqrt{3}}{\left(x-\varphi_{1}\right)^{2}+4\left(t-\varphi_{2}\right)^{2}-2 \sqrt{3}\left(t-\varphi_{2}\right)+1} e^{2 i t} .
\end{aligned}
$$

For $\varphi_{1,2}=0$ the plot of $|v(x, t)|$ is presented in Fig. 1 .

More precisely, we obtain the original Peregrine solution introduced in Peregrine (1983) by setting $\varphi_{1}=0$ and $\varphi_{2}=$ $\frac{\sqrt{3}}{4}$.

The case $n=2$ where we have 4 phases $\left(\varphi_{1,2,3,4}\right)$ is already much more interesting: it provides the first example of 3 rogue waves solutions of the NLS equation and, under a very special selection of phases, reproduces the simplest "higher order" Peregrine breather first found in Akhmediev et al. (1985). Below we present the plots of the $|v(x, t)|$ corresponding to $B=1$ and to some particular selections of phases for $n=2,3,4$ making the multiple rogue waves character evident in the solutions described by the main theorem above. The denominator of the rational part of the solution is a 6-th order polynomial with respect to $x$ and $t$.

Because of the length of the related expression, we provide here only a graphic representation of $|v(x, t)|$ (see Fig. 2). The detailed "polynomial" formula for these solutions is given in Appendix A. The phases $\varphi_{1}$ and $\varphi_{2}$ are again translation parameters, but $\varphi_{3}$ and $\varphi_{4}$ have more influence on the behavior of the solutions.

We can consider these solutions as a 4-parametric extension of the second-order solution given in Akhmediev et al. (2009a). The later is distinguished by the presence of one highest maximum and 4 additional smaller maxima, contrary

\footnotetext{
${ }^{2}$ For the proof see Dubard et al. (2010); Eleonskii et al. (1986), although the proofs and notations are slightly different and a proof in Dubard et al. (2010) is shorter and simpler.
} 


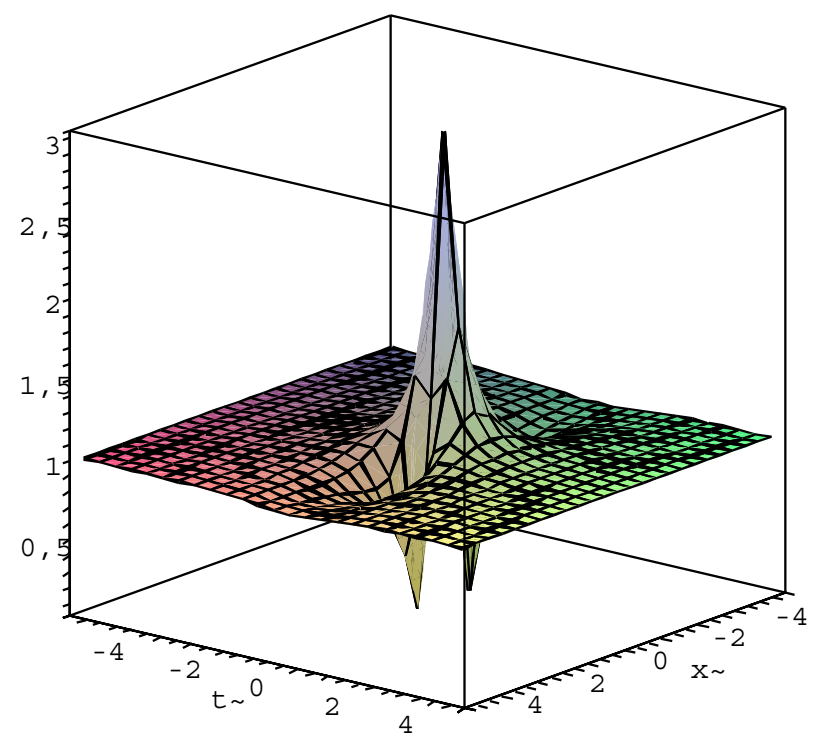

Fig. 1. Plot of $|v(x, t)|$ for $n=1, \varphi_{1}=0$ and $\varphi_{2}=0$.

to the behavior of the solutions corresponding to the generic choice of phases. It corresponds to the following specific selection of the phases:

$\varphi_{1}=\varphi_{3}=0$,

$\varphi_{2}=\frac{(7+2 \sqrt{5}) \sqrt{10-2 \sqrt{5}}}{24}$,

$\varphi_{4}=\frac{(5+\sqrt{5}) \sqrt{10-2 \sqrt{5}}}{96}$.

The related plot of $|v(x, t)|$ is given by the Fig. 3 .

In general, the denominator of the rational part of the solution is a $n(n+1)$-th order polynomial with respect to $x$ and $t$. For the generic choice of the phases, the solution seems to have $n(n+1) / 2$ maxima and $n(n+1)$ minima. An appropriate specific choice of parameters should allow the appearance of "super-peak(s)". Figure 4 presents the solutions obtained with all phases equal to 0 in the cases $n=3$ and $n=4$.

\section{From multi-rogue waves solutions of NLS equation to KP-I equation}

Here we apply the previous results to the KP-I equation:

$\partial_{x}\left(4 u_{t}+6 u u_{x}+u_{x x x}\right)=3 u_{y y}$.

Denote $g(x, y, t)$ the function

$g(x, y, t):=\left.v\left(x, t, \varphi_{3}\right)\right|_{t=y, \varphi_{3}=t}$.
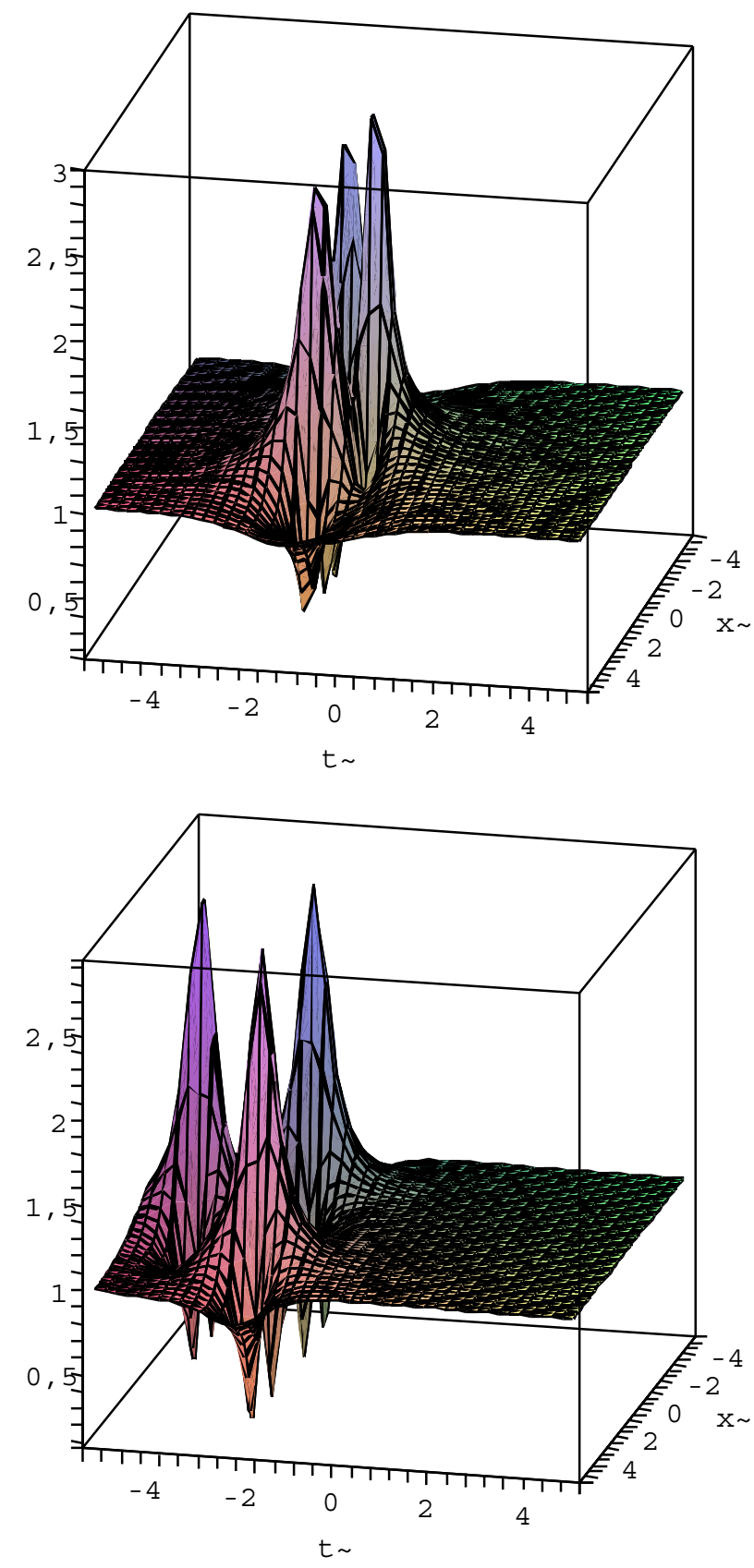

Fig. 2. Plot of $|v(x, t)|$ for $n=2$ with $\varphi_{1}=\varphi_{2}=\varphi_{3}=\varphi_{4}=0$ at the top and $\varphi_{4}=1$ and $\varphi_{1}=\varphi_{2}=\varphi_{3}=0$ at the bottom.

In other words $g$ is obtained by replacing the independent variable $t$ in $v(x, t)$ by $y$ and the phase $\varphi_{3}$ by $t$. The following theorem relates the described class of quasi-rational solutions of the NLS equation to a $2 n$ parametric family of smooth localized real rational solutions of the KP-I equation. Its most important part - formula (6) - was first proved by in (Dubard et al., 2010; Matveev et al., 2010). 


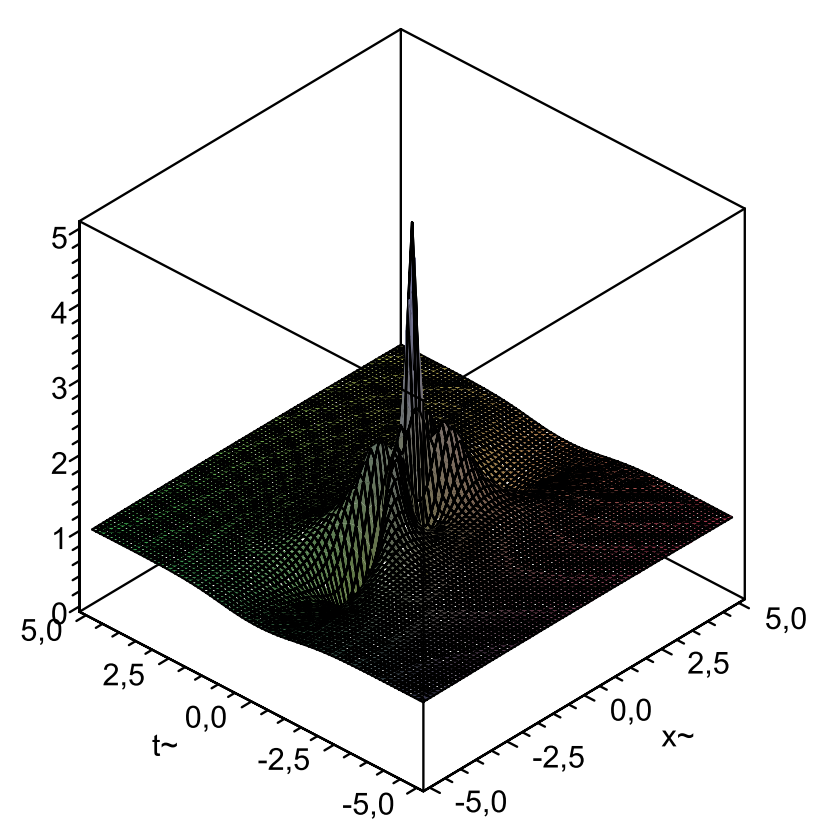

Fig. 3. Amplitude of the Peregrine breather of order 2.

Theorem 2. The function

$u(x, y, t):=2\left(|g|^{2}-B^{2}\right)=\left.2 \partial_{x}^{2} \log W_{1}\right|_{t=y, \varphi_{3}=t}$

is a smooth, localized real rational solution to the KP-I equation, satisfying the relations

$\int_{-\infty}^{\infty} u(x, y, t) d x=0$,

and

$\forall t, \quad u(x, y, t) \rightarrow 0, \quad$ when $\quad x^{2}+y^{2} \rightarrow \infty$

Therefore, any plot of the amplitude of the solution (3) corresponds to the plot of the solution to the KP-I equation at the moment of time $t=\varphi_{3}$. The related maxima of the solutions of the KP-I equation become more sharp, since with respect to the NLS case, they are described by a square of the amplitude of the solution of the NLS equation shifted down on the constant $-B^{2}$. It is also evident that for some special moments of time, the confluence of the KProgue waves takes place corresponding to the "higher order" Peregrine breathers. At such moments of time, the solution of the KP-I equation reaches its highest possible value which decays afterwards. We believe that this is a very first explicit manifestation of the 2-D rogue waves described by the precise analytic expression. This also gives a new view and physical interpretation of the "higher order" Peregrine breathers as describing a relief of a two-dimensional rogue wave, (described by the KP-I equation) at the moment when its height reaches the absolute maximum.
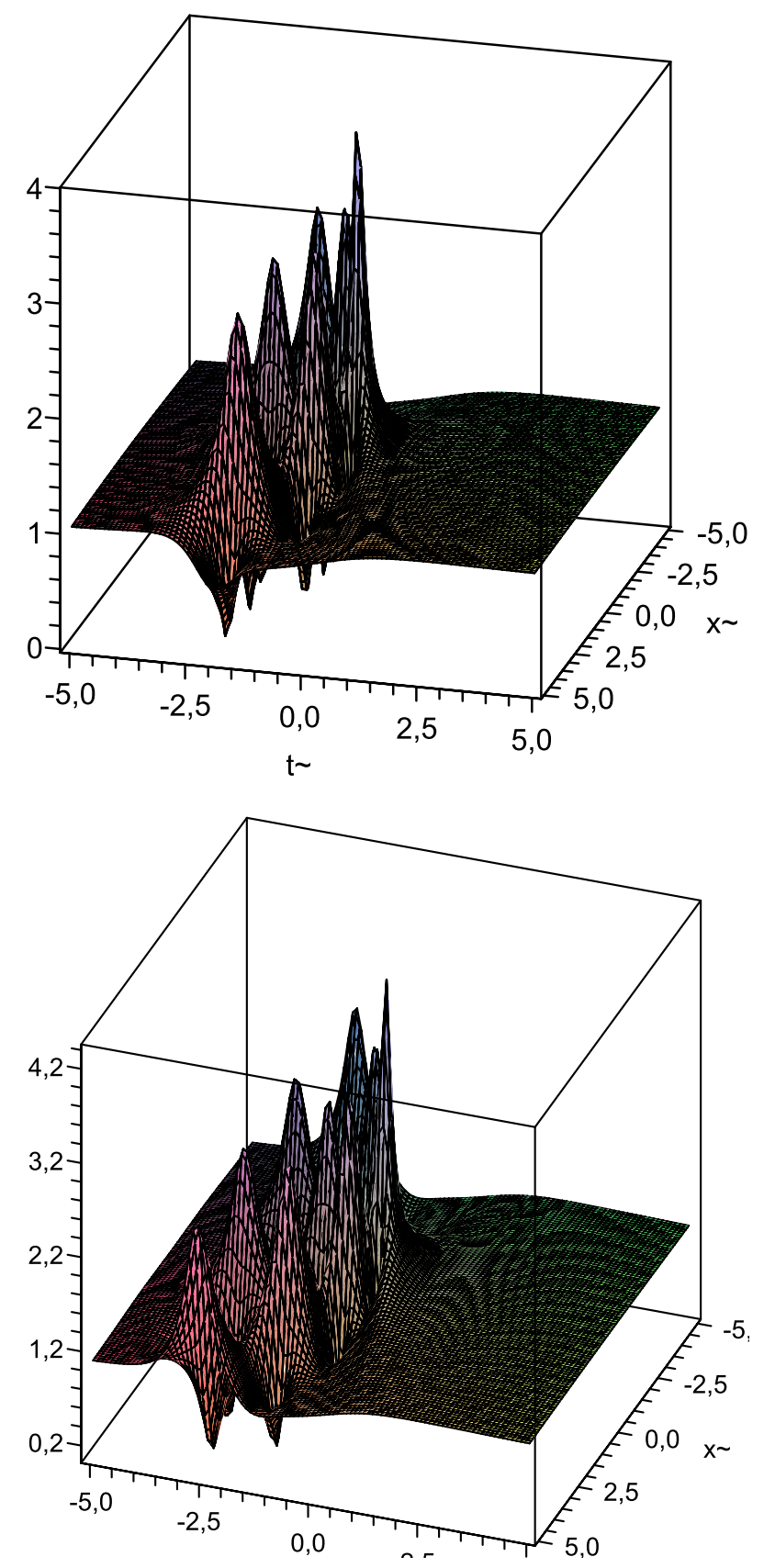

Fig. 4. Plot of $|v(x, t)|$ with vanishing phases for $n=3$ at the top and $n=4$ at the bottom.

\section{Concluding remarks}

- The plots quite similar to ours but corresponding to the "pre-rogue" waves solutions periodic in $x$ and observed inside one period can be found in Calini (2002); Schober (2006). From the oceanographic point of view these pre-rogue wave solutions appear less realistic since, due to different factors (wind, dissipation, dispersion, etc.), it is difficult to imagine the 
appearance of the true infinite $x$-periodic train of rogue waves. In fiber optics the situation is quite different. In fact, observations made recently in Kibler et al. (2010) concern not the pure Peregrine breather, but the aforementioned "pre-rogue" waves solutions being very close to the Peregrine breather for a sufficiently big value of the period parameter. See also Shrira (2010) for further useful comments.

- For more general multi-periodic elementary solutions describing the $\mathrm{N}$-periodic modulation of the plane wave solution, closed analytical expressions were obtained in Belokolos et al. (1994), Its et al. (1988). In principle, passage to the limit when all periods tend to infinity should also produce the quasi-rational solutions to the focusing NLS equation, but actually this has been achieved by A. R. Its, private communication, June 2010, only for obtaining the second order "higher" Peregrine breather and technically also seems to be rather involved.

- An advantage of our approach is its possibility to explore the results obtained for the NLS equation to get interesting new results for the KP-I equation explained above. In fact, the correspondence between the solutions of the NLS equation and the KP-I equation might be extended to the whole class of the so called finite-gap multi-periodic solutions, yet we are avoiding discussing this topic here in order to preserve the elementary level of our presentation.

\section{Appendix}

The solution presented below is a special case of (3) with $n=2$. It corresponds to the choice of parameters giving the easiest form for the numerical evaluation and producing the plots of the solutions. Below we set $B=1$ and

$\varphi_{1}:=3 \varphi_{3}, \quad \varphi_{2}:=2 \varphi_{4}+\frac{3+\sqrt{5}}{16} \sqrt{10-2 \sqrt{5}}$.

The whole family of solutions with $n=2$ can be obtained by a scaling transformation and space and time translations. The related solution $v(x, t)$ depends on two parameters $\alpha$ and $\beta$ proportional to $\varphi_{3}, \varphi_{4}$ :

$\alpha:=(5+\sqrt{5}) \sqrt{10-2 \sqrt{5}}-96 \varphi_{4}, \quad \beta:=96 \varphi_{3}$.

It reads

$v(x, t)=\left(1-12 \frac{G(2 x, 4 t)+i H(2 x, 4 t)}{Q(2 x, 4 t)}\right) e^{2 i t}$,

where

$$
\begin{aligned}
G(x, t):= & x^{4}+6 g_{2}(t) x^{2}+2 \beta x+g_{0}(t), \\
H(x, t):= & t x^{4}+2 h_{2}(t) x^{2}+2 \beta t x+h_{0}(t), \\
Q(x, t):= & x^{6}+3 g_{2}(t) x^{4}-2 \beta x^{3}+3 q_{2}(t) x^{2} \\
& +6 \beta g_{2}(t) x+q_{0}(t)
\end{aligned}
$$

with

$$
\begin{aligned}
& g_{2}(t):=t^{2}+1, \\
& g_{0}(t):=5 t^{4}+18 t^{2}-4 \alpha t-3, \\
& h_{2}(t):=t^{3}-3 t+\alpha, \\
& h_{0}(t):=t^{5}+2 t^{3}-2 \alpha t^{2}-15 t+2 \alpha, \\
& q_{2}(t):=t^{4}-6 t^{2}+4 \alpha t+9, \\
& q_{0}(t):=t^{6}+27 t^{4}-4 \alpha t^{3}+99 t^{2}-36 \alpha t+\beta^{2}+4 \alpha^{2}+9 .
\end{aligned}
$$

The Peregrine breather of order 2, the same as first found in Akhmediev et al. (1985), whose amplitude is plotted in Fig. 3, is obtained when $\alpha=\beta=0$.

Acknowledgements. This work has been supported partly by the grant ANR-09-BLAN-0117-01.

We wish to thank J. Dudley and B. Kibler for very useful explanations concerning their work (Kibler et al., 2010) and the anonymous referees for the useful remarks.

Edited by: E. Pelinovsky

Reviewed by: two anonymous referees

\section{References}

Akhmediev, N., Eleonskii, V. Z., and Kulagin, N.: Generation of periodic trains of picosecond pulses in an optical fiber: exact solutions, Sov. Phys. JETP-USSR, 62, 894-899, 1985.

Akhmediev, N., Ankiewicz, A., and Soto-Crespo, J. M.: Rogue waves and rational solutions of the nonlinear Schrödinger, Phys. Rev. E, 80, 026601, doi:10.1103/PhysRevE.80.026601, 2009a.

Akhmediev, N., Ankiewicz, A., and Taki, M.: Waves that appear from nowhere and disappear without a trace, Phys. Lett. A, 373, 675-678, 2009b.

Akhmediev, N., Soto-Crespo, J. M., and Ankiewicz, A.: Extreme waves that appear from nowhere: on the nature of rogue waves, Phys. Lett. A, 373, 2137-2145, 2009c.

Ankiewicz, A., Clarkson, P. A., and Akhmediev, N.: Rogue Waves, rational solutions, the patterns of their zeros and integral relations, J. Phys. A-Math. Theor., 43, 122002, doi:10.1088/1751-8113/43/12/122002, 2010.

Belokolos, E. D., Bobenko, A. I., Enol'ski, V. Z., Its, A. R., and Matveev, V. B.: Algebro-geometric approach to nonlinear integrable equations, Springer-Verlag, Series in nonlinear dynamics, 1-337, 1994.

Bordag, L., Its, A., Matveev, V., Manakov, S., and Zakharov, V.: Two-dimensional solitons of the Kadomtsev-Petviashvily equation and their interaction, Phys. Lett. A, 63, 205-206, 1977.

Calini, A. and Schober, C. M.: Homoclinic chaos increases the likelyhood of rogue wave formation, Phys. Lett. A, 298, 335349, 2002.

Dubard, P., Gaillard, P., Klein, C., and Matveev, V. B.: On multirogue wave solutions of the NLS equation and positon solutions of the KdV equation, Eur. Phys. J. Special Topics, 185, 247-258, doi:10.1140/epjst/e2010-01252-9, 2010.

Eleonskii, V., Krichever, I., and Kulagin, N.: Rational multisoliton solutions to the NLS equation, Sov. Dokl. Math. Phys., 287, 606$610,1986$. 
Kibler, B., Fatome, J., Finot, C., Millot, G., Dias, F., Genty, G., Akhmediev, N., and Dudley, J. M.: The Peregrine Solution in Nonlinear Fibre Optics, Nat. Phys., 6, 790-795, doi:10.1038/nphys 1740, 2010.

Its, A. R., Rybin, A. V., and Salle, M. A.: Exact Integration of Nonlinear Schrödinger equation, Theor. Math. Phys., 74, 29-45, 1988.

Kharif, C., Pelinovsky, E., and Slunyaev, A.: Rogue Waves in the Ocean, Springer-Verlag, 2009.

Matveev, V. B. and Salle, M. A.: Darboux Transformations and solitons, in: Series in Nonlinear Dynamics, Springer-Verlag, 1991.
Matveev, V. B. and Dubard, P.: The constructions of extremal wave solutions for some integrable systems, in: Proc. of the Int. Conf. FNP 2010 Frontiers of Nonlinear Physics, Nijni Novgorod - StPetersburg, 06-13-2010, 100-101, 2010.

Peregrine, D. H.: Water waves, nonlinear Schrödinger equations and their solutions, J. Aust. Math. Soc. B, 25, 16-43, 1983.

Schober, C. M.: Melnikov analysis and inverse spectral analysis of rogue waves in deep water, Eur. J. Mech. B-Fluid., 25, 602-620, 2006.

Shrira, V. and Geogjaev, V.: What makes the Peregrine soliton so special as a prototype of freak waves?, J. Eng. Math., 67, 11-22, 2010.

Zhdanov, S. and Trubnikov, B.: Soliton chains in a plasma with magnetic viscosity, ZHETF Pis'ma v Redaktsiiu, 39(3), 110$113,1984$. 